\title{
The game to promote the capability of reducing, reuse and recycling waste
}

\author{
Elsa Rodrigues,", and Luís Bruno \\ School of Technology and Management (ESTG)- Laboratory of Systems of Information and Interactivity (LabSI2) \\ Polytechnic Institute of Beja, Rua Pedro Soares, Beja, Portugal
}

\begin{abstract}
This work aims to raise awareness of environmental problems through interactive means, which, today, are much more appealing than the traditional ones to the academic community. To better inform community members of the importance of the 3Rs - Reduce, Reuse and Recycle, it was decided to develop an interactive 3D game, accessible through the Word Wide Web. This game includes 3 different activities which can be played in any order: solid waste recycling, saving water, and saving electricity. A small group of people evaluated the games; all participants were familiar with video games. It should be noted that the tests carried out focused only on the usability and interactivity of the game - the impact of the game at the 3Rs level - Reduce, Reuse and Recycle - has not been tested yet. The game makes the user more aware of the physical space where he moves (the school), showing his progress in real time. At the end of the game, the user is presented with the total time he took to finish his tasks and the total score achieved. In this way, the game becomes more interesting to the user.
\end{abstract}

\section{Introduction}

This essay is based on the Eco-Schools project at the School of Technology and Management (ESTIG) of the Polytechnic Institute of Beja. ESTIG joined the EcoSchools program in December 2015. This is an international program of the Foundation for Environmental Education, developed in Portugal since 1996 by the Blue Flag Association of Europe (ABAE) [1]. It aims to encourage actions and recognize the quality of the work developed by the school within the subject of Environmental Education for Sustainability.

ESTIG's first Eco-Schools council brought together students, teachers, school staff, and other entities (Quercus, Beja City Council, League for Nature Protection, Alentejo Public Waters), which discussed the following themes: water, waste, energy, and healthy and sustainable food. It was decided to audit the use of the following resources: water, waste (plastic, glass and paper), energy, mobility, and food. For this game, only water, waste and energy are taken into account.

In this context, ESTIG has developed a structured quality project considering a better management and environmental education of the members of the school as well as a greater awareness of changing attitudes, behaviors and citizenship, in order to improve the quality of everyday life at school. This essay focuses only on water, solid waste and energy resources.

To better inform community members of the importance of the 3Rs - Reduce, Reuse and Recycle -, it was decided to develop an interactive 3D game, accessible through the Word Wide Web. The game takes place in a 3D virtual representation of the ESTIG building. This game includes 3 different activities which can be played in any order: solid waste recycling, saving water and saving electricity.

\subsection{Objectives of the Game}

In today's society, it is increasingly important to make humans aware of their responsibility to contribute to the sustainability of planet Earth. To this end, campaigns can be conducted through the media, associations, among others. Thus, ESTIG decided to contribute to this campaign by raising the academic community's environmental awareness through a 3D game called "3Rs".

The main objective of the game is to make the population aware of the importance of saving water, electricity and waste collection (paper, glass and plastic). Thus, the game consists of these 3 activities, which take place in ESTIG's virtual model.

\subsection{Process and scope of research}

The project encompassed five phases. This process was centered on the users [2]. The first phase of the project was based on the analysis of functional and nonfunctional requirements of the system, which allowed characterizing the actors that intervene in the system.

*Corresponding author: elsa.rodrigues@ipbeja.pt 
The second stage corresponded to the design process, in which scenarios of use were created. The third phase consisted in the creation of the prototype and the fourth phase focused on the evaluation of the prototype and this involved making necessary changes. The evaluation tests were performed with the users, which allowed us to evaluate the usability of the game.

\section{Analysis and Design of the game}

This chapter covers user analysis, system requirements, which define user requirements for the computer system to be developed, and describes functional and nonfunctional requirements. Functional requirements describe system functions and services, while nonfunctional requirements describe constraints on which the system must operate (operating systems, time constraints) and be developed (specific languages) [3].

We will present the usage (activity) scenarios that illustrate the system to be used by users to perform the identified actions, allowing us to understand how the tasks will be performed, thus creating the screen design that will address two interrelated purposes: functionality and aesthetics [2], [4] and [5]. In terms of functionality, a well-designed screen makes information clear and helps users understand how they can interact with it. In aesthetic terms, a well-designed screen is attractive, attracting users' attention, implying the creation of low, medium and high fidelity prototypes.

\subsection{Analysis}

\subsubsection{Functional and non-functional requirements and general system requirements}

The analysis of functional and non-functional requirements is the first phase of software development. For the process of development of any type of software, it is essential that the system requirements (functional and non-functional) are well defined. The functional requirements define the functionality of a system, while non-functional requirements define system properties and/or restrictions on the services or functions provided by it [3].

In this case, the functional requirements are: nonlinear gameplay, because the player can play any of the games without a predefined order; player ranking, which allows you to view your score throughout the game. The non-functional requirements are: persistence and consistency data, which are related to the reliability of software that covers accuracy and data consistency; compatibility, device independence - allows software to run on different systems; usability, which relates the specification of interfaces and user interactions with the system, focusing on consistent interfaces. The measurable attribute for usability requirements use was likeability (which denotes 'ease of use'). The most straightforward way to measure user satisfaction is through customer inquiries.

However, the general system requirements are: to complete the objective of the game; to inform and sensitize the player to a real world problem; to go through various stages of the game in different spaces of ESTIG; to show energy saving and recycled waste graphs. Furthermore, the system was implemented in Unity $3 D$, executable on a web platform; the user can only use the mouse and the keyboard.

\subsubsection{Design of the interfaces}

The design of the system is very important because it represents the reality with which the user deals with his daily life [6]. As such, when carrying out this phase, we considered interface design and screen design. The interface design includes: 1) choice of interaction objects (text boxes, buttons, etc.); 2) Screen design (Find objects on screen, logical groupings of objects ...); 3) Design of navigation in the application; 4) Design of interaction with the context (Applications, OS, physical environment).

While in screen design, we must ask what to do. As such, we had to ask some questions, such as: 1) What operations should the user do?; 2) What information, order, comparisons, etc. are required to perform the operations?

In that way, we define low-fidelity prototypes, medium-prototypes and high-fidelity prototypes. Therefore, we take into account Human Centered Design Processes for Interactive System (DCU) as defined in ISO 13407, [5] and [6], one of its main objectives being to define a correct allocation of roles between the system and the user, which implies the active involvement of users. In this sense, we perform the analysis of users and tasks, through the user scenarios, which we describe.

For the "recycling" game, the following usage scenario was created [6], [7]: Maria starts the game at the entrance of ESTIG. At the start of the game, Maria is presented with the different games as well as their goals and game controls. After reading the objectives and instructions, Maria decides to go to the $\mathrm{H} 2 \mathrm{O}$ room, where the Recycling game takes place. Maria knows she has to put the garbage, which is scattered in the $\mathrm{H} 2 \mathrm{O}$ room and in the courtyard, in the respective Eco point. They have to press the E key when they are near objects. Therefore, Maria grabs the empty plastic bottle using the "E" key and goes to the Eco point where she places the bottle in the yellow bag and earns 100 points. Now, Maria grabs the glass bottle and places it in the green bag and earns 100 more points. Continuing to collect the trash, Maria grabs the sheets of paper and places them in 
the blue bag and earns another 100 points. This completes the task with 300 points and she is notified that the task of this game has been completed. However, it is up to her which games she has yet to complete and where they are located.

For the water saving game, following usage scenario was created [6], [7]: Eduardo starts the game at the entrance of ESTIG, chooses one of 3 different games. The game shows the objectives and controls. Eduardo decides to go to the men's toilet in the basement (floor 1), where the water saving game takes place. Arriving in the bathroom, Eduardo knows he needs to close the 3 open taps. To do this, he must stand in front of each tap and allow the "E key" to close them, earning 100 points for each closed tap. He has to repeat the process until all three taps are closed. After the tap is closed, he will be notified that he has completed the tasks for this game and will see the unfinished games and where they are located.

For the "electricity saving" game, the following scenario was developed [6], [7]: Teresa starts the game at ESTIG's entrance, where she is presented with the different games she can play, as well as her goals and game controls. Teresa decides to go to Lab12, where the electricity saving game takes place. Teresa knows that in order to finish the game, she needs to turn off the 4 monitors. Teresa stands in front of the first monitor and presses the "E" key to turn it off and earns 100 points. Teresa repeats the process for the other monitors. When all monitors are off, Teresa is notified that she has completed the game and, at the same time, she is informed of the remaining unfinished games and where they are located.

After having developed the usage scenarios, we created the prototypes, which are a partial representation of the system we intend to develop.

A prototype can be anything from a drawing on a piece of paper to a representation on a screen or set of screen or even a three-dimensional model, a set of screenshots or a software extract. The prototypes are divided into 3 groups: low-fidelity, medium-fidelity and high-fidelity prototypes (these after implementation) [3].

At this point, the low and medium-fidelity prototypes defined for the game will be presented. These represent the main activities.

The two first medium-prototypes are for the "recycling" game in the $\mathrm{H} 2 \mathrm{O}$ living room (fig. 1) and in ESTIG's terrace (fig. 2).

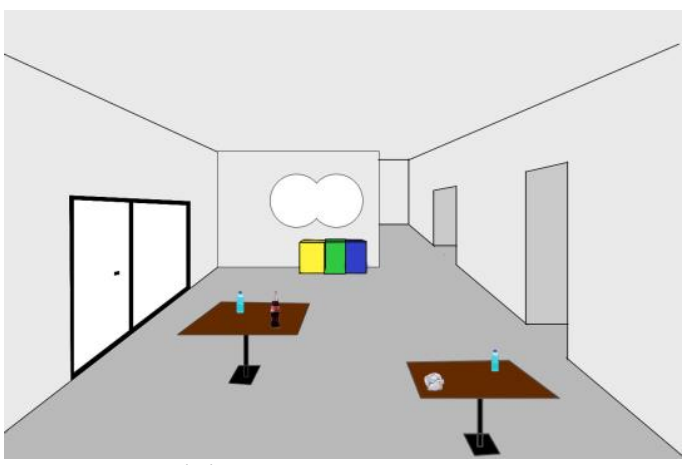

Fig. 1. H2O Living Room.

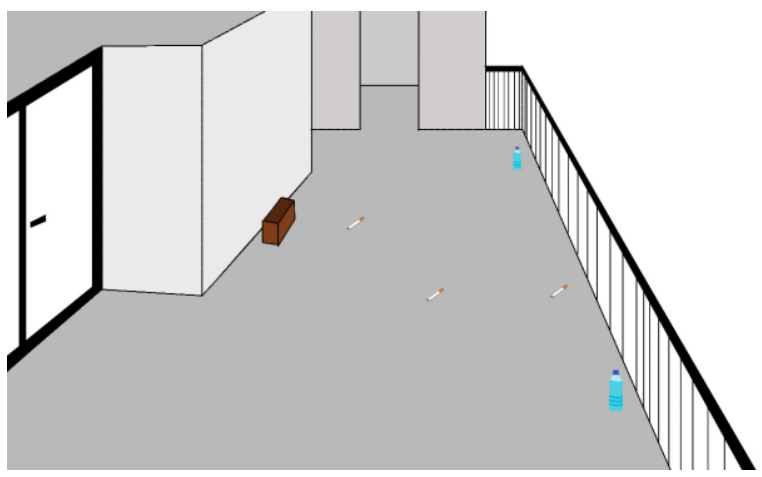

Fig. 2. ESTIG's terrace.

The third medium prototype is for the game "save electricity", which takes place in Lab12 (fig. 3).

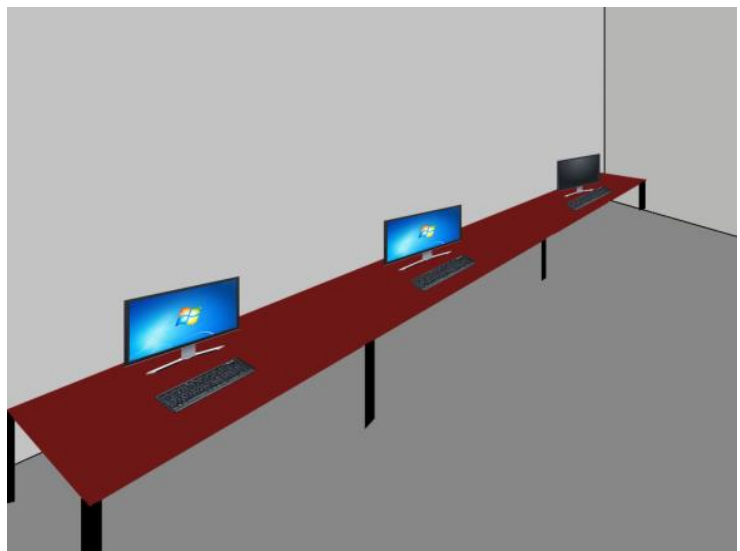

Fig. 3. Classroom Lab12 (floor -1).

We will now introduce the low-fidelity prototype concerning the water saving game, which takes place in the men's bathroom in the basement (floor -1) (fig. 4). 


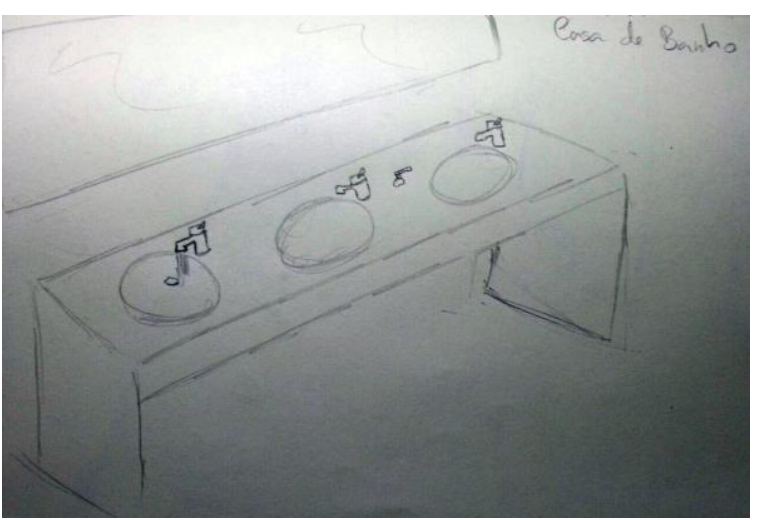

Fig. 4. Men's bathroom (floor -1).

After this, it was necessary to define the controls of the game, which will be described in the next subsection.

\subsubsection{Controls of the game}

Controls define the interface of a game that typically uses the keyboard or mouse. The simplicity of game controls are essential for game quality and acceptance [4] or other gaming devices. In our case, the controls we have used were the mouse and keyboard, with the following functions: Mouse: used to control the camera; W, A, S, D keys: used to control the player's movement; E key: used to interact with the various objects; TAB key: used to show / hide the objectives; Shift key: run; Space key: jump; Enter key: used to restart the game.

\section{Development and tests of the game}

The software development process corresponds to a set of partially ordered activities aimed at obtaining a software product. [3] The test phase is of key relevance for its sucess and aims to detect errors in the systems so they can be corrected in advance. Tests must be carried out during software development, as the earlier an error is detected, the lower the cost associated with its correction will be [8]. One can even say that tests are related to quality. As stated by Craig and Jaskiel "Testing is a concurrent lifecycle process of engineering, using and maintaining testware in order to measure and improve the quality of the software being tested" [9].

\subsection{Development}

The technologies used were Unity3D, C \# and Photoshop. Unity 3D was chosen because it is software that enables game development. It also makes the programmer's job easier, as he does not need to program directly for DirectX or OpenGL as it is automatic. Unity lets you create games for Apple products (Mac, iPhone, iPod, iPad), Microsoft (Xbox, Windows), Google (Android devices), Sony (PlayStation 3), Nintendo (Wii), and Web browsers (Internet Explorer, Mozilla Firefox, Google Chrome, Opera and Safari) [10]. The interface is quite user-friendly. It has a Mono-based scripting tool (a tool for developing and running .NET applications on different platforms), enabling $\mathrm{C}$ \#, UnityScript, and Bool programming. Photoshop is a graphic design and digital imaging software that is available for Microsoft Windows and Mac OS X operating systems, and Unity3D supports various image and sound files such as Photoshop, MP3, OGG, WAV, among others [11].

The implementation was made by 3 students of the Computer Engineering course. This implementation was based on work previously done by a student of the same course and was developed for mobile platforms (in Unity3D and C \#) which work with the help of virtual reality glasses. Thus, it was necessary to convert the game controls for the use of the keyboard and mouse.

For the "recycling" game, we considered the different types of waste and associated them with the corresponding types of (containers). The $\mathrm{C} \#$ scripts created for the implementation of this game allow the player to pick up the trash on the floor and to place it in the corresponding eco-points. If placed in the right ecopoints, they receive a positive score, otherwise negative. The following is the high-fidelity prototype of the $\mathrm{H} 2 \mathrm{O}$ living room (fig. 5).

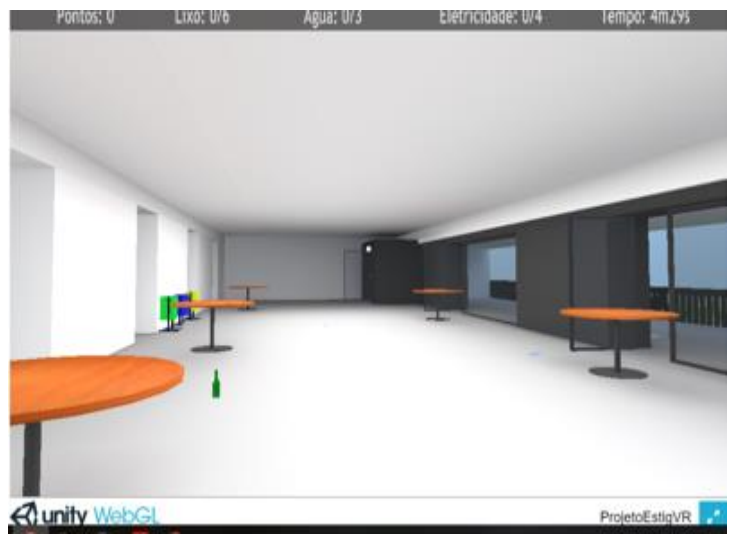

Fig. 5. H2O Living Room.

We present the high-fidelity prototype of the recycling at the terrace, see fig. 6 . 


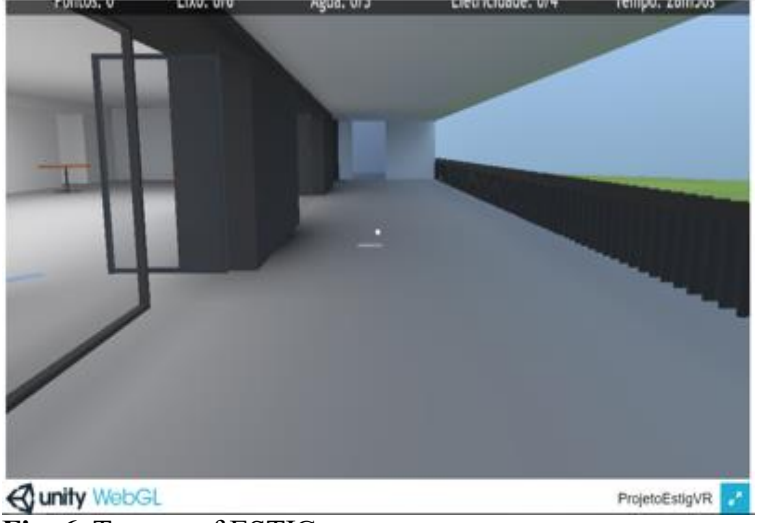

Fig. 6. Terrace of ESTIG

For the "save electricity" game, it was necessary to do the 3D modeling of the computers in Lab12, the room where the game takes place. The C \# script created for the implementation allows the player to interact with the monitors. For this action to be possible, the player has to position himself in front of the monitor. You can see the high-fidelity prototype of classroom Lab12 in fig. 7.

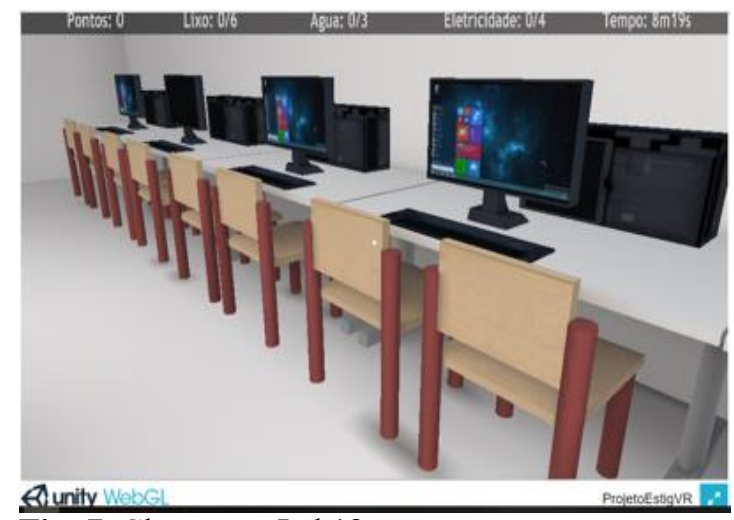

Fig. 7. Classroom Lab12

To implement the water saving game, it was necessary to do the 3D modeling of the men's bathroom as well as to create the $3 \mathrm{D}$ particle system that represents water. The $\mathrm{C} \#$ script created allows the player to interact with the open water taps; for that he needs to be in front of the water taps. The figure below depicts the highfidelity prototype of the men's bathroom (fig. 8).

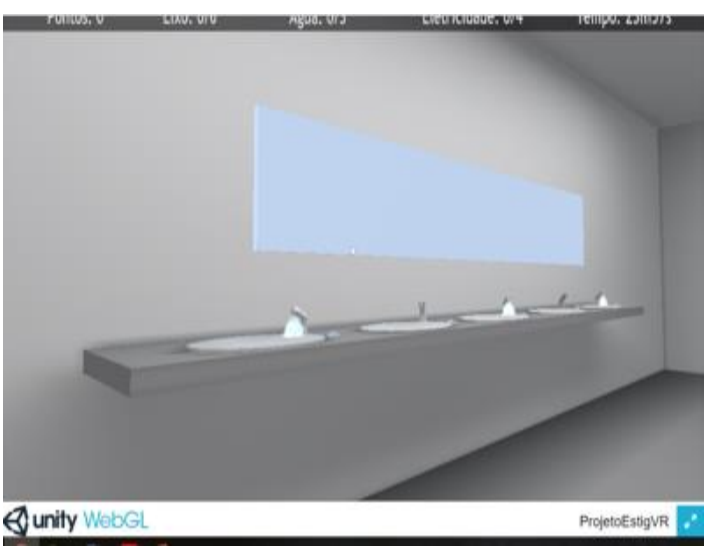

Fig. 8. Men's bathroom
During the implementation process, several team meetings were held (with teachers and students), which allowed the development of the scoring system of all games, showing the player their progress in real time, the areas where game activities are taking place, the objectives of the games and their rules are shown, and which or which games have not been finished. When the player finishes all games, the time used and the total points are shown; besides this information, the player has the option to restart the game. It is important to bear in mind that the game was optimized to run on the web, so it was necessary to recreate and optimize the game's light system. Thus, WebGL bluid works seamlessly in a web environment.

\subsection{Tests with players}

The tests are very important in the software development process [3], [8]. In this way, gameplay and usability tests were performed with the users who served to test the gaming experience allowing us to identify the components that contributed the most to the experience and to find a balance in the parameters, such as sharpening the tension of the experience and the difficulty curve [12]. The users who participated in the tests belong to the game's intended audience and did not participate in the development process. They were computer engineering students, used to the gaming platform; we can put them in the hardcore gamer group. Table 1 describe the questions of the test with the users.

Table 1. Game evaluation.

\begin{tabular}{|c|}
\hline Scale from 0 (very bad) to 5 (Excellent) \\
\hline 1. How realistic is ESTIG's virtual building model? \\
\hline 2. How realistic are the integrated objects? \\
\hline $\begin{array}{l}\text { 3. What is the level of control of the user's position in the } \\
\text { building? }\end{array}$ \\
\hline $\begin{array}{l}\text { 4. What is the level of control of grasping and dropping } \\
\text { objects to recycle? }\end{array}$ \\
\hline 5. What is the control level of closing the water taps? \\
\hline 6 . What is the control level of turning off the screens? \\
\hline $\begin{array}{l}\text { 7. Do you consider this game important in education } \\
\text { for recycling and saving water and electricity? }\end{array}$ \\
\hline 8. Comments and proposals for improvement? \\
\hline
\end{tabular}


COMPUTER TECHNICAL CHARACTERISTICS

1. What browser is used?

2. Environment indication (Windows / MAC / others) or Operating System?

3. Computer Memory

4. Computer Graphics Card.

As can be seen from table 1, we have divided the game classification into 2 groups. The first group focuses on the characteristics of the game, the second group focuses on the characteristics of the computer. Twenty-three students participated in the test. Graph 1 shows the answers from the first group to question 7.

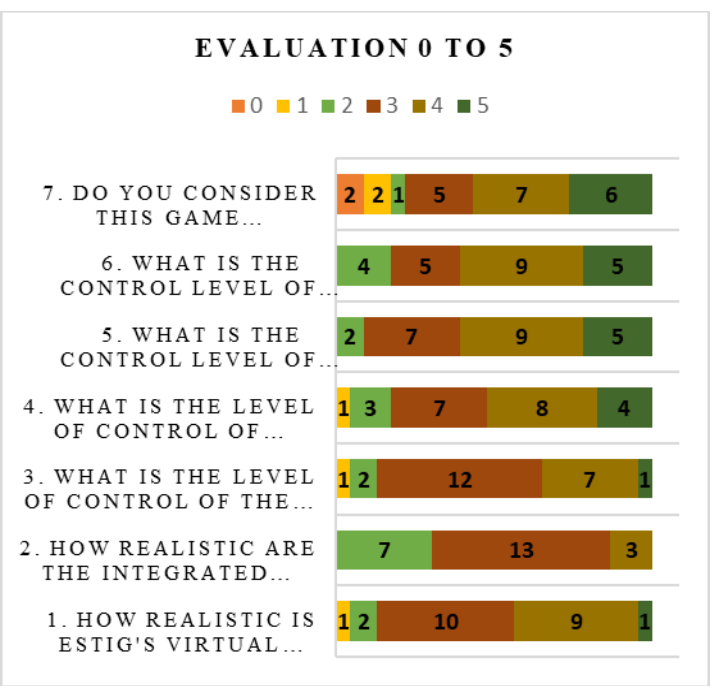

Graph 1. Results of game evaluation.

For question 8, most students did not suggest any improvements. However, some students suggested the following improvements: the inclusion of the school's elevator; greater specificity in interactions, such as pointing to the computer button to shut down; creating the button to exit the game; more visible objects (difficulty distinguishing whether glass is plastic or glass); very bright white; very bright colors; the field of view should be changed to zoom out; need to improve graphics; the menu of suggestions and objectives should be more interactive; need to improve mouse sensitivity; creation of a short tutorial.

The questions of the second group are about the computer technical characteristics. Just one student has a computer with the Linux system, the others have windows 10. The browsers used are Google Chrome, Firefox, Opera. Participants' computer memory ranges from $4 \mathrm{~GB}$ to $32 \mathrm{~GB}$; the information about Computer Graphics Cards is very diverse.

By analyzing the test results, we found that there are features that could be improved, such as: the level of control of the user's position in the building; the virtual building model; objects should be altered to make them more realistic and to allow a more accurate differentiation.

\section{Conclusions and future work}

We conclude that the game has very particular features as it was developed specifically for ESTIG's virtual environment. The game allows the user to get a better sense of the school's physical space, allows the game to be played randomly, ie, in the order the user wishes, shows the user their progress in real time, and at the end of the game the user receives information on the time spent playing the game and on the total score achieved. This makes the game interesting for the user. However, the objective of contributing to water and electricity saving and to the dissemination of recycling practices has not been fully achieved; moreover, the answers to questions 1 and 2 were not the expected ones.

As future work and this according to the tests, we found that the presentation of the graphics and some objects should be improved, the controls must also be more precise. In addition, the virtual environment should be even more real.

\section{Acknowledgements}

Publication is the result of participation in the project Organization of the 9th International Scientific and Technical Conference entitled Environmental Engineering, Photogrammetry, Geoinformatics - Modern Technologies and Development Perspectives" funded by the Polish National Agency for Academic Exchange under the International Academic Partnerships Programme.

\section{References}

1 A. -. A. B. A. d. Europa, "Eco-Escolas," [Online]. Available: https://ecoescolas.abae.pt/sobre/quemsomos/ (2018)

2 L. H. Shawn, "Notes on User Centered Design Process (UCD)," (31 03 2004). [Online]. Available: https://www.w3.org/WAI/redesign/ucd

3 I. Sommerville, Software Engineering, 10 ed., England: Pearson Education Limited, 2015.

4 J. B. Vasconcelos e N. Ribeiro, Tecnologias de Programação de jogos, Lisboa: FCA - editora de Informática, Lda., (2013).

5 M. J. Fonseca, P. Campos e D. Gonçalves, Introdução ao design de interfaces, Lisboa: FCA Editora de Informática, Lda, (2017).

6 B. Shneiderman, Designing the user interface, $3 \mathrm{rd}$ ed., Addison-Wesley, (1998).

7 J. Grudin, "Personas: Practice and Theory," [Online]. 
Available:

http://research.microsoft.com/users/jgrudin/publicati ons/personas/Pruitt-Grudin.pdf. [Acedido em (2019)].

8 G. J.Myers, The Arts of Software Testing, New Jersey: John Wiley \& Sons, Inc, (2004).

9 R. D. Craig e S. P. Jaskiel, Systematic Software Testing, London: Artech House Publishers, (2002).

10 Unity, "Unity Documentation," [Online]. Available: https://docs.unity3d.com/Manual/systemrequirements.html. [Acedido em (2018)].

11 Unity, "Unity for all," [Online]. Available: https://unity.com/. [Acedido em (2018)].

12 J. Herbert e A. Price, "Métodos para Avaliação da Qualidade de Software," em XIV Congresso da Sociedade Brasileira de Computação, Porto Alegre, (1995). 